\title{
Ultraluminous X-ray sources
}

\author{
Kirill Atapin ${ }^{* 1,2}$ \\ ${ }^{1}$ Sternberg Astronomical Institute, Moscow State University, Universitetsky pr., 13, Moscow, \\ 119991, Russia \\ 2 Special Astrophysical Observatory, Nizhnij Arkhyz, Zelenchukskiy region, \\ Karachai-Cherkessian Republic, 369167, Russia \\ E-mail: atapin.kirill@gmail.com
}

\begin{abstract}
Ultraluminous X-ray sources (ULXs) represent a class of binary systems that are more luminous than any black hole in our Galaxy. The nature of these objects remained unclear for a long time. The most popular models for the ULXs involve either intermediate mass black holes (IMBHs) or stellar-mass black holes accreting at super-Eddington rates. In the last few years our understating of these objects was significantly improved, which made the model of super-Eddington accretion much preferable. Both the X-ray and optical spectra provide evidence for the strong outflows coming from supercritical accretion disk. Another surprising result was discovery of pulsations in four ULXs, which claims that these systems must host neutron stars. Besides the presence of pulsations, there is no sharp difference between ultraluminous pulsars and normal ULXs. This fact implies that significant number of known ULXs might eventually be neutron stars.
\end{abstract}

Accretion Processes in Cosmic Sources - II - APCS2018

3-8 September 2018

Saint Petersburg, Russian Federation

${ }^{*}$ Speaker. 


\section{Introduction}

Ultraluminous X-ray sources are point-like extragalactic sources with X-ray luminosity above the Eddington limit for normal stellar mass black hole $\left(\sim 10^{39} \mathrm{erg} / \mathrm{s}\right)$. They are assumed to be not associated with galactic nuclei or background quasars and cannot be powered by accretion onto supermassive black holes. So this definition establishes a class of objects that are more luminous, assuming isotropic emission, than X-ray binaries observed in the Milky Way. First ULXs were discovered by Einstein observatory [1, 2, 3], which first had sufficient angular resolution in X-rays to distinguish them from active galactic nuclei. At present a few hundreds of ULXs are known [4, 5]. Most of them are located in star-forming galaxies and associated with young stellar population $[6,7,8,9]$.

Two basic models have been proposed to explain the ULX phenomenon. First of them involves black holes with masses of 1000-10000 $\mathrm{M}_{\odot}$ [10], so-called intermediate-mass black holes (IMBH). Since the Eddington limit depends on the mass, in the case of IMBH observed luminosities should correspond to sub-critical accretion with $L \sim 0.1-0.01 L_{E d d}$. This accretion regime is typical for Galactic black hole binaries (GBHBs), so IMBH model implies that ULXs might be massive analogues of GBHBs and could show similar properties [11, 12]. Possible scenario of IMBH formation involves remnants of the Population III stars [13] or runaway merging in dense clusters [14].

Another model is a super-Eddington accretion (with or without beaming) onto stellar-mass objects [15, 16, 17]. A concept of supercritical disks was proposed by Shakura and Sunyaev [18] and further developed in works [19, 20, 21, 17, 22]. A key feature of the super-Eddington accretion predicted by theory and reproduced by MHD-simulations [23, 24] is a strong optically thick wind, which covers the inner parts of the disc and collimates the radiation. In this scenario ULXs may be similar to Galactic superaccretor SS 433 [25, 26]. This system is powered by Roche-lobe accretion from an evolved A-type supergiant donor star, shows strong optically-thick outflow together with semi-relativistic baryonic jets [26]. Apparent X-ray luminosity of SS 433 is about $10^{36} \mathrm{erg} / \mathrm{s}$ [27] due to high inclination, however, being observed nearly face-on orientation this system might be look like a ULX [15].

At present community has come to consensus that most of the ULXs are more likely to be superaccretors. This is became evident after the absorption lines of the wind had been directly observed in grating spectra of some ULXs [28, 29]. Moreover, at least four ULXs turned out to be pulsating neutron stars [30, 31, 32,33]. This fact implies that Eddington limit could be exceeded by hundred times. Nevertheless, the most extreme ULXs with luminosity up to $10^{42} \mathrm{erg} / \mathrm{s}$ (so-called hyperluminous X-ray sources, e.g. ESO 243-49 [34]) can not be explained by super-Eddington accretion and remain good candidates for IMBHs.

\section{Spectral and variability properties}

As first high-quality observations from XMM-Newton and Chandra appear, it became clear that spectra of ULXs differ from those observed in GBHBs. They demonstrate unambiguous curvature above $2 \mathrm{keV}[35,36]$ which cannot be fitted by power law and hints at cutoff at higher energies. This cutoff is located outside the energy range of XMM-Newton and Chandra but it was 
eventually confirmed by NuStar [37, 38]. Being fitted by standard for GBHBs model consisting of a multi-color disk and Comptonized corona, the ULX spectra yield colder disks and much colder and thicker Comptonizing medium than seen in GBHBs $\left(k T_{\text {disk }} \sim 0.2 \mathrm{keV}, k T_{e} \sim 1-2 \mathrm{keV}\right.$ and $\tau \gtrsim 6$ in ULXs [36] versus $k T_{\text {disk }} \sim 1 \mathrm{keV}, k T_{e} \sim 100 \mathrm{keV}$ and $\tau \lesssim 1$ in GBHBs [39]). This spectral shape unusual for GBHBs, introduced was introduced as new, 'ultraluminous' accretion state [36].

Depending on the contribution of the high- and low-energy parts of the spectrum, the ultraluminous state can be splitted into three classes [40]: soft ultraluminous (SUL), hard ultraluminous (HUL) and broadened disc (BD). The last one shows most prominent curvature which can be formally fitted by single disk component with temperature of 1-2.5 keV. There is a tendency for a particular source to belong to a specific class, although transitions between classes have also been observed [41]. It was found that BD-type spectrum is usually shown by less luminous ULXs, while SUL and HUL sources equally bright [40]. On the other hand, when a particular SUL or HUL source becomes brighter during its spectral evolution, it shows more curved BD-like spectrum $[42,41,43]$.

Another class of sources neighboring to ULXs is ultraluminous supersoft sources (ULSs). Their spectra are dominated by black-body component with temperature $\sim 100 \mathrm{eV}$, and there is almost no emission above $\sim 1 \mathrm{keV}[44,45]$. These sources are not easy to study because the maximum of their emission falls into inaccessible far-UV range. ULSs may be considered as subclass of ULXs because some sources are observed to jump from the supersoft state to the soft ultraluminous $[46,47,29]$.

Lines in the spectra of ULXs have long been the subject of intense search. Originally absorption lines and edges produced by the wind have been predicted for ULXs on the grounds of SS $433[48,49]$. Latter they were clearly suspected in the highest-quality spectra of NGC 5408 X-1, NGC 6946 X-1 [50] and NGC 1313 X-1 [51]. Eventually blue-shifted absorptions with velocities $v \sim 0.2 c$ together with emission lines at rest have been discovered in RGS spectra of same sources [28] and also in NGC 55 ULX [29] and NGC 300 ULX-1 [52]. Interestingly, that the last one is ultraluminous pulsar (see below), while sources NGC 5408 X-1, NGC 1313 X-1 and NGC 55 ULX can be classified as SUL, HUL and ULS respectively [40, 29]. So, one can conclude that the presence of the outflow does not depend on the source spectral type.

Ultraluminous X-ray sources demonstrate strong stochastic variability at different time scales. Some of them change their flux by tens $[53,41]$ and even hundred times $[54,55]$ on time scales of month-years. Short-term variability ( $\sim 1$ hour) is generally higher in the SUL sources [56, 40], however most of this variability is contributed by the hard component of their spectra [57, 40]. In $1-10 \mathrm{keV}$ energy band the fractional rms variability of the SUL sources reaches $40 \%$ [40]. ULSs are even more variable. They exhibit dips - sharp drops of the flux on a timescale of a few hundreds of seconds [29, 47].

Typical power spectra may be described by power law or broken power law [56]. Five ULXs M82 X-1, NGC 5408 X-1, NGC 6946 X-1, NGC 1313 X-1 and IC 342 X-1 - show quasi-periodic oscillations with frequencies from 0.01 to $0.6 \mathrm{~Hz}[58,59,60,61,62]$. The QPO frequency is found to be negatively correlated with the fractional variability $[63,64]$ and positively with the source luminosity [64]. Also for sources NGC 5408 X-1 and NGC6946 X-1 a linear relation between the absolute rms variability and flux has been reveled $[65,66]$. A linear rms-flux relation is common for all accreting systems from active galactic nuclei to cataclysmic stars [67, 68, 69]. 


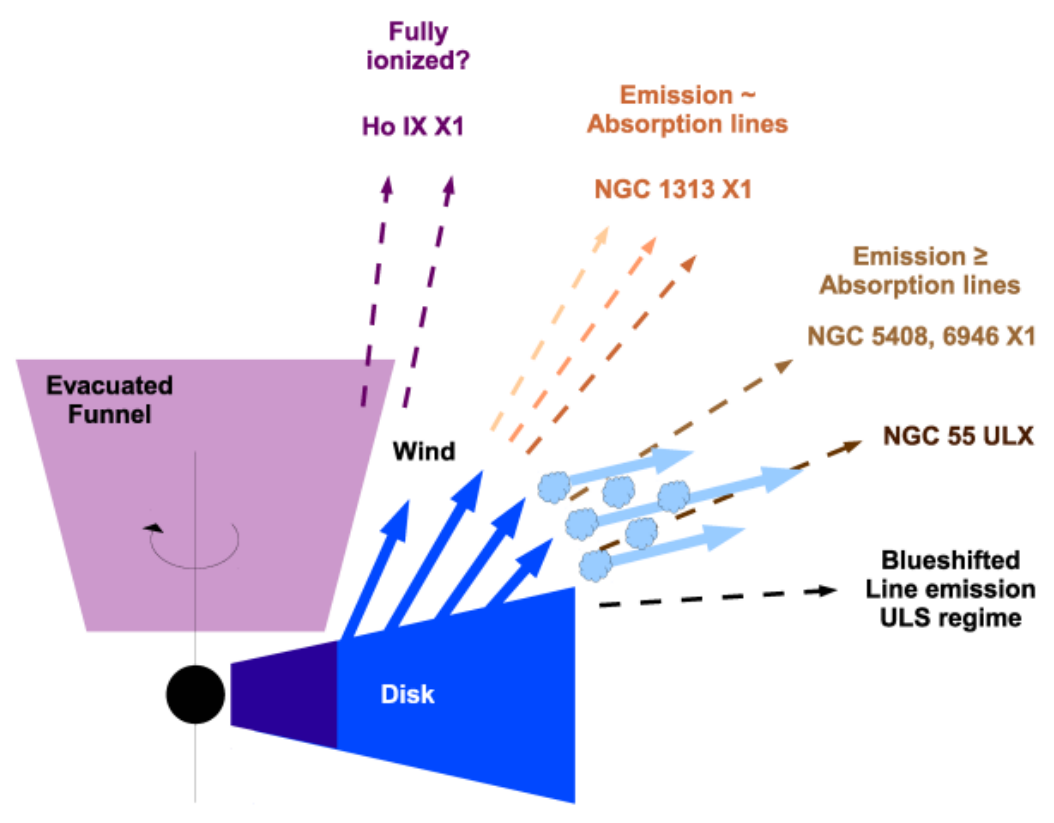

Figure 1: Schematic representation of the supercritical accretion disc illustrating relation between the type of observed spectrum and the disk inclination angle, taken from [29].

Currently there is still no model that could unify all diversity of spectral and variability properties of ULXs. It has been proposed that, besides the accretion rate, the important role in that what makes the sources different, may be played by inclination of the supercritical accretion disk [57, 40, 70]. This idea is illustrated in Fig. 1. Numerical MHD-simulations showed that wind is not isotopic, it looks like a funnel with some opening angle [23, 24]. If the source is viewed nearly along the funnel axis, observer could directly see the innermost parts of the accretion disc emitting hard X-rays; such a source would be classified as HUL. At higher inclination the wind can intersect line of sight, and the observed spectrum would be dominated be soft thermal component (SUL). As the inclination increases, the optical depth of the wind also increases, and when the inclination reaches some critical angle, the wind could completely block the hard emission (ULS). Probably, increasing inclination even more, one would see SS433-like object [15], totally obscured in X-rays (observed X-ray luminosity of SS433 is only $0.1 \%$ of its total power [27]).

The IMBH candidate ESO 243-49 HLX-1 stands away from other ULXs. Its behavior is quite similar to that seen in GBHBs. This source undergoes repeating outbursts and show spectra consistent with canonical spectral states [34, 71]. Assuming that at the outbursts maxima the source is in the thermal state, one can obtain the black hole mass. It is between 3000 and $3 \times 10^{5} \mathrm{M}_{\odot}$ depending on spin parameter [72, 71].

\section{Optical counterparts}

Most of the ULXs are located in crowded fields which hampers a firm identification of their optical counterparts. Although X-ray catalogs contain $\sim 500$ ULXs, unique point-like optical counterparts are known only for $\sim 20$ objects $[73,74,75]$. All of them are relatively faint sources with 
$m_{V} \gtrsim 21$. The distribution of the absolute magnitudes has a sharp maximum at $M_{V} \approx-6$ [76]. The brightest sources NGC 6946 X-1 and NGC 7793 P-13 (the last one is ultraluminous pulsar, see below) have magnitude $M_{V} \approx-7.5$ [77, 78] (SS 433 even brighter, $M_{v} \approx-8$ [26]). For the magnitudes $M_{V} \gtrsim-5$ the number of objects rapidly decreases which can be related both to the effects of observational selection and to the physical reasons [76].

Only the faintest optical counterparts show star-like spectral energy distributions (SEDs) [55, 76]. These SEDs being classified as spectra of F- or G-type supergiants may be interpreted as emission of the donor stars. Other counterparts demonstrate power-law SEDs with maximum in UV band $[74,77]$. This fact might be considered as an evidence for the hot optically thick outflow irradiated by hard emission from the innermost parts of the disk [77].

About 10 optical counterparts have been studied spectrally [79, 80, 81, 76]. All of them show similar optical spectra resembling the spectra of WNL or LBV stars and also SS 433 [81]. The spectra have broad He II $\lambda 4686$ and hydrogen $\mathrm{H} \alpha$ and $\mathrm{H} \beta$ emission lines with widths ranging from 500 to $1500 \mathrm{~km} / \mathrm{s}$. It has been shown that these lines cannot originate from the donor star or irradiated disk, and must be produced by accelerated wind [81]. In the case of irradiated disk, the He II lines should be broader than Balmer lines because the former are produced in the inner disk regions rotating more rapidly. This effect is observed in GBHBs [82, 83] but in ULXs we have an opposite situation. In the case of radiation-pressure driven wind, the Balmer lines should be broader, since they comes from the regions of wind located further from the source and already gained speed.

\section{Ultraluminous $X$-ray pulsars}

The most exciting discovery of last years was the detection of coherent pulsations in ultraluminous X-ray source M82 X-2 [30]. After that pulsations were discovered also in sources NGC 7793 P13 [31, 84], NGC 5907 ULX [32] and NGC 300 ULX-1 [33]. The presence of pulsations claims that these systems must host neutron stars. The pulsation periods are between $0.42 \mathrm{~s}$ (NGC 7793 P13) and $\approx 30 \mathrm{~s}$ (NGC $300 \mathrm{ULX}-1)$, the pulsation fraction is about $20-30 \%$ in all the objects. The most luminous pulsar is NGC 5907 ULX. Its peak luminosity reaches $10^{41} \mathrm{erg} / \mathrm{s}$, which apparently exceeds the Eddington limit for a neutron star by $\sim 500$ times and limiting luminosity of X-ray pulsars [85] by $\sim 100$ times . Many models involving magnetic field and beaming of different strength have been proposed to solve this problem [86, 87, 88, 89, 32].

How many neutron stars are among known ULXs? Besides the presence of pulsations, properties of ultraluminous pulsar is seems to be quite usual for ULXs. The spectra of ULX-pulsars are harder but they still can be classified as the HUL or BD state [90] and fitted by can be the same models [91]. Pulsar NGC 300 X-1 shows outflow with the same velocity as the nonpulsing-ULXs. Apparently, the detection of pulsation remains the only reliable criterion to distinguish neutron start one from black holes. Although an analysis of the wide sample of well-studied ULXs did not detect pulsations [92], on the other hand, pulsations are also absent in many observations of confirmed ULX-pulsars. So, the question is still open.

Probably, the answer to this question depends on how extreme conditions are required for neutron star to reach the ULX luminosity. Population synthesis showed that neutron stars are 1050 times more numerous than black holes[93]. However, if it requires magnetic fields of huge 
strength [89] or very strong collimation of radiation, the number of neutron stars among ULXs may be not high.

\section{Conclusions}

Ultraluminous X-ray sources represent a divisive population. Objects with low and medium luminosity $10^{39}<L_{X}<10^{41} \mathrm{erg} / \mathrm{s}$ are more likely neutron stars and stellar mass black holes accreting at super-Eddington rates, with increasing number of neutron stars closer to low-luminosity edge. Hyperluminous sources are probably intermediate mass black holes accreting at sub-Eddington regime. At present, we have no reliable instruments to determine the nature of the accretor in each particular nonpulsing-ULX. But this instrument may appear in the future, as the ULX-pulsar will be studied more properly.

Acknowledgements The research was supported by the Russian Science Foundation grant N 14-50-00043 and RFBR grant N 18-32-20214.

\section{References}

[1] K. S. Long, S. Dodorico, P. A. Charles and M. A. Dopita, Observations of the X-ray sources in the nearby SC galaxy M33, ApJL 246 (1981) L61.

[2] D. J. Helfand, Endpoints of stellar evolution - X-ray surveys of the Local Group, PASP 96 (1984) 913.

[3] G. Fabbiano, X rays from normal galaxies, ARA\&A 27 (1989) 87.

[4] D. A. Swartz, K. K. Ghosh, A. F. Tennant and K. Wu, The Ultraluminous X-Ray Source Population from the Chandra Archive of Galaxies, ApJS 154 (2004) 519 [astro-ph/ 04054 98].

[5] D. J. Walton, T. P. Roberts, S. Mateos and V. Heard, 2XMM ultraluminous X-ray source candidates in nearby galaxies, MNRAS 416 (2011) 1844 [1106.0197].

[6] G. Fabbiano, A. Zezas and S. S. Murray, Chandra Observations of "The Antennae" Galaxies (NGC 4038/9), ApJ 554 (2001) 1035 [astro-ph/ 0102256 ].

[7] T. P. Roberts, R. S. Warwick, M. J. Ward and S. S. Murray, A Chandra observation of the interacting pair of galaxies NGC 4485/4490, MNRAS 337 (2002) 677 [astro-ph/ 0208196 ].

[8] D. A. Swartz, A. F. Tennant and R. Soria, Ultraluminous X-Ray Source Correlations with Star-Forming Regions, ApJ 703 (2009) 159 [0907.4718].

[9] J. Poutanen, S. Fabrika, A. F. Valeev, O. Sholukhova and J. Greiner, On the association of the ultraluminous $X$-ray sources in the Antennae galaxies with young stellar clusters, MNRAS 432 (2013) $506[1210.1210]$.

[10] E. J. M. Colbert and R. F. Mushotzky, The Nature of Accreting Black Holes in Nearby Galaxy Nuclei, ApJ 519 (1999) 89 [astro-ph/9901023].

[11] P. Kaaret, A. H. Prestwich, A. Zezas, S. S. Murray, D.-W. Kim, R. E. Kilgard et al., Chandra High-Resolution Camera observations of the luminous X-ray source in the starburst galaxy M82, MNRAS 321 (2001) L29 [astro-ph/ 0009211$].$

[12] J. M. Miller, G. Fabbiano, M. C. Miller and A. C. Fabian, X-Ray Spectroscopic Evidence for Intermediate-Mass Black Holes: Cool Accretion Disks in Two Ultraluminous X-Ray Sources, ApJL 585 (2003) L37 [astro-ph/ 0211178 ]. 
[13] P. Madau and M. J. Rees, Massive Black Holes as Population III Remnants, ApJL 551 (2001) L27 [astro-ph/0101223].

[14] S. F. Portegies Zwart, H. Baumgardt, P. Hut, J. Makino and S. L. W. McMillan, Formation of massive black holes through runaway collisions in dense young star clusters, Nature 428 (2004) 724 [astro-ph/0402622].

[15] S. Fabrika and A. Mescheryakov, Face-on SS 433 stars as a possible new type of extragalactic X-ray sources, in Galaxies and their Constituents at the Highest Angular Resolutions (R. T. Schilizzi, ed.), vol. 205 of IAU Symposium, p. 268, Jan., 2001, a stro-ph/ 0103070.

[16] A. R. King, M. B. Davies, M. J. Ward, G. Fabbiano and M. Elvis, Ultraluminous X-Ray Sources in External Galaxies, ApJL 552 (2001) L109 [astro-ph / 0104333 ].

[17] J. Poutanen, G. Lipunova, S. Fabrika, A. G. Butkevich and P. Abolmasov, Supercritically accreting stellar mass black holes as ultraluminous X-ray sources, MNRAS 377 (2007) 1187 [astro-ph/0609274].

[18] N. I. Shakura and R. A. Sunyaev, Black holes in binary systems. Observational appearance., A\&A 24 (1973) 337.

[19] M. A. Abramowicz, M. Calvani and L. Nobili, Thick accretion disks with super-Eddington luminosities, ApJ 242 (1980) 772.

[20] M. A. Abramowicz, B. Czerny, J. P. Lasota and E. Szuszkiewicz, Slim accretion disks, ApJ 332 (1988) 646.

[21] G. V. Lipunova, Supercritical disk accretion with mass loss, Astronomy Letters 25 (1999) 508 [astro-ph/9906324].

[22] C. Dotan and N. J. Shaviv, Super-Eddington slim accretion discs with winds, MNRAS 413 (2011) 1623 [1004.1797].

[23] K. Ohsuga, M. Mori, T. Nakamoto and S. Mineshige, Supercritical Accretion Flows around Black Holes: Two-dimensional, Radiation Pressure-dominated Disks with Photon Trapping, ApJ 628 (2005) 368 [arXiv:astro-ph/0504168].

[24] K. Ohsuga and S. Mineshige, Global Structure of Three Distinct Accretion Flows and Outflows around Black Holes from Two-dimensional Radiation-magnetohydrodynamic Simulations, ApJ 736 (2011) 2 [1105.5474].

[25] S. N. Fabrika, The Supercritical Accretion Disk of SS 433, Ap\&SS 252 (1997) 439.

[26] S. Fabrika, The jets and supercritical accretion disk in SS433, ASPRv 12 (2004) 1 [arXiv:astro-ph/0603390].

[27] A. Medvedev and S. Fabrika, Evidence of supercritical disc funnel radiation in X-ray spectra of SS 433, MNRAS 402 (2010) 479 [0910.3186].

[28] C. Pinto, M. J. Middleton and A. C. Fabian, Resolved atomic lines reveal outflows in two ultraluminous X-ray sources, Nature 533 (2016) 64 [1604.08593].

[29] C. Pinto, W. Alston, R. Soria, M. J. Middleton, D. J. Walton, A. D. Sutton et al., From ultraluminous $X$-ray sources to ultraluminous supersoft sources: NGC 55 ULX, the missing link, MNRAS 468 (2017) 2865 [1612.05569].

[30] M. Bachetti, F. A. Harrison, D. J. Walton, B. W. Grefenstette, D. Chakrabarty, F. Fürst et al., An ultraluminous X-ray source powered by an accreting neutron star, Nature 514 (2014) 202 [1410.3590]. 
[31] F. Fürst, D. J. Walton, F. A. Harrison, D. Stern, D. Barret, M. Brightman et al., Discovery of Coherent Pulsations from the Ultraluminous X-Ray Source NGC 7793 P13, ApJL 831 (2016) L14 [1609.07129].

[32] G. L. Israel, A. Belfiore, L. Stella, P. Esposito, P. Casella, A. De Luca et al., ULX-1 in NGC5907: how bright can an accreting pulsar shine?, ArXiv e-prints (2016) [1609.07375].

[33] S. Carpano, F. Haberl, C. Maitra and G. Vasilopoulos, Discovery of pulsations from NGC 300 ULX1 and its fast period evolution, MNRAS 476 (2018) L45 [1802 .10341].

[34] S. A. Farrell, N. A. Webb, D. Barret, O. Godet and J. M. Rodrigues, An intermediate-mass black hole of over 500 solar masses in the galaxy ESO243-49, Nature 460 (2009) 73 [1 001 . 0567].

[35] A.-M. Stobbart, T. P. Roberts and J. Wilms, XMM-Newton observations of the brightest ultraluminous X-ray sources, MNRAS 368 (2006) 397 [astro-ph/ 0601651 ].

[36] J. C. Gladstone, T. P. Roberts and C. Done, The ultraluminous state, MNRAS 397 (2009) 1836 [0905.4076].

[37] M. Bachetti, V. Rana, D. J. Walton, D. Barret, F. A. Harrison, S. E. Boggs et al., The Ultraluminous X-Ray Sources NGC 1313 X-1 and X-2: A Broadband Study with NuSTAR and XMM-Newton, ApJ 778 (2013) 163 [1310.0745].

[38] D. J. Walton, F. A. Harrison, B. W. Grefenstette, J. M. Miller, M. Bachetti, D. Barret et al., Broadband $X$-Ray Spectra of the Ultraluminous X-Ray Source Holmberg IX X-1 Observed with NuSTAR, XMM-Newton, and Suzaku, ApJ 793 (2014) 21 [1402.2992].

[39] J. E. McClintock and R. A. Remillard, Black hole binaries, pp. 157-213. Apr., 2006.

[40] A. D. Sutton, T. P. Roberts and M. J. Middleton, The ultraluminous state revisited: fractional variability and spectral shape as diagnostics of super-Eddington accretion, MNRAS 435 (2013) 1758 [1307.8044].

[41] F. Pintore, L. Zampieri, A. Wolter and T. Belloni, Ultraluminous X-ray sources: a deeper insight into their spectral evolution, MNRAS 439 (2014) 3461 [1401. 6815].

[42] F. Pintore and L. Zampieri, X-ray spectral states and metallicity in the ultraluminous X-ray sources NGC 1313 X-1 and X-2, MNRAS 420 (2012) 1107 [1110.6277].

[43] W. Luangtip, T. P. Roberts and C. Done, The X-ray spectral evolution of the ultraluminous X-ray source Holmberg IX X-1, MNRAS 460 (2016) 4417 [1605. 08246].

[44] J. Liu and R. Di Stefano, An Ultraluminous Supersoft X-Ray Source in M81: An Intermediate-Mass Black Hole?, ApJL 674 (2008) L73 [0 802 . 0 507].

[45] R. Soria and A. Kong, Revisiting the ultraluminous supersoft source in M 101: an optically thick outflow model, MNRAS 456 (2016) 1837 [1511.04797].

[46] R. Urquhart and R. Soria, Optically thick outflows in ultraluminous supersoft sources, MNRAS 456 (2016) 1859 [1511.05275].

[47] H. Feng, L. Tao, P. Kaaret and F. Grisé, Nature of the Soft ULX in NGC 247: Super-Eddington Outflow and Transition between the Supersoft and Soft Ultraluminous Regimes, ApJ 831 (2016) 117 [1608.07212].

[48] S. Fabrika, S. Karpov, P. Abolmasov and O. Sholukhova, Properties of SS433 and ultraluminous $X$-ray sources in external galaxies, in Populations of High Energy Sources in Galaxies (E. J. A. Meurs and G. Fabbiano, eds.), vol. 230 of IAU Symposium, pp. 278-281, 2006, a stro-ph/0510491, DOI. 
[49] S. N. Fabrika, P. K. Abolmasov and S. Karpov, The supercritical accretion disk in SS 433 and ultraluminous X-ray sources, in Black Holes from Stars to Galaxies - Across the Range of Masses (V. Karas and G. Matt, eds.), vol. 238 of IAU Symposium, pp. 225-228, Apr., 2007, astro-ph/0610664, DOI.

[50] M. J. Middleton, D. J. Walton, T. P. Roberts and L. Heil, Broad absorption features in wind-dominated ultraluminous X-ray sources?, MNRAS 438 (2014) L51 [1309. 3134].

[51] M. J. Middleton, D. J. Walton, A. Fabian, T. P. Roberts, L. Heil, C. Pinto et al., Diagnosing the accretion flow in ultraluminous $X$-ray sources using soft X-ray atomic features, MNRAS 454 (2015) $3134[1509.06760]$.

[52] P. Kosec, C. Pinto, D. J. Walton, A. C. Fabian, M. Bachetti, M. Brightman et al., Evidence for a variable Ultrafast Outflow in the newly discovered Ultraluminous Pulsar NGC 300 ULX-1, MNRAS 479 (2018) 3978 [1803.02367].

[53] F. Grisé, P. Kaaret, H. Feng, J. J. E. Kajava and S. A. Farrell, X-ray Spectral State is not Correlated with Luminosity in Holmberg II X-1, ApJL 724 (2010) L148 [1011.2231].

[54] M. Brorby, P. Kaaret and H. Feng, Transition of an X-ray binary to the hard ultraluminous state in the blue compact dwarf galaxy VII Zw 403, MNRAS 448 (2015) 3374 [1502. 03443 ].

[55] S. Avdan, A. Vinokurov, S. Fabrika, K. Atapin, H. Avdan, A. Akyuz et al., Optical counterparts of two ULXs in NGC 5474 and NGC 3627 (M 66), MNRAS 455 (2016) L91 [1510 . 07915 ].

[56] L. M. Heil, S. Vaughan and T. P. Roberts, A systematic study of variability in a sample of ultraluminous X-ray sources, MNRAS 397 (2009) 1061 [0 905.1265 ].

[57] M. J. Middleton, T. P. Roberts, C. Done and F. E. Jackson, Challenging times: a re-analysis of NGC 5408 X-1, MNRAS 411 (2011) 644 [1009.2686].

[58] T. E. Strohmayer and R. F. Mushotzky, Discovery of X-Ray Quasi-periodic Oscillations from an Ultraluminous X-Ray Source in M82: Evidence against Beaming, ApJL 586 (2003) L61 [astro-ph/0303665].

[59] T. E. Strohmayer, R. F. Mushotzky, L. Winter, R. Soria, P. Uttley and M. Cropper, Quasi-periodic Variability in NGC 5408 X-1, ApJ 660 (2007) 580 [astro-ph/ 0701390 ].

[60] F. Rao, H. Feng and P. Kaaret, Detection of Strong Short-term Variability in NGC 6946 X-1, ApJ 722 (2010) 620 [1008.2065].

[61] D. R. Pasham, S. B. Cenko, A. Zoghbi, R. F. Mushotzky, J. Miller and F. Tombesi, Evidence for High-frequency QPOs with a 3:2 Frequency Ratio from a 5000 Solar Mass Black Hole, ApJL 811 (2015) L11 [1601.02628].

[62] V. K. Agrawal and A. Nandi, Discovery of a quasi-periodic oscillation in the ultraluminous X-ray source IC 342 X-1: XMM-Newton results, MNRAS 446 (2015) 3926 [1411. 4135].

[63] B. De Marco, G. Ponti, G. Miniutti, T. Belloni, M. Cappi, M. Dadina et al., Time lags in the ultraluminous X-ray source NGC 5408 X-1: implications for the black hole mass, MNRAS 436 (2013) 3782 [1310.0042].

[64] K. Atapin, S. Fabrika, M. D. Caballero-García, Ultraluminous X-ray sources with flat-topped noise and $Q P O, M N R A S$ (2019), in press.

[65] L. M. Heil and S. Vaughan, The linear rms-flux relation in an ultraluminous X-ray source, MNRAS 405 (2010) L86 [1 003.5825$].$ 
[66] L. Hernández-García, S. Vaughan, T. P. Roberts and M. Middleton, X-ray time lags and non-linear variability in the ultraluminous X-ray sources NGC 5408 X-1 and NGC 6946 X-1, MNRAS 453 (2015) 2877 [1508.01328].

[67] P. Uttley and I. M. McHardy, The flux-dependent amplitude of broadband noise variability in X-ray binaries and active galaxies, MNRAS 323 (2001) L26 [astro-ph/ 0103367 ].

[68] S. Vaughan, A. C. Fabian and K. Nandra, X-ray continuum variability of MCG-6-30-15, MNRAS 339 (2003) 1237 [astro-ph/0211421].

[69] S. Scaringi, E. Körding, P. Uttley, P. J. Groot, C. Knigge, M. Still et al., Broad-band timing properties of the accreting white dwarf MV Lyrae, MNRAS 427 (2012) 3396 [1208 . 6292].

[70] M. J. Middleton, L. Heil, F. Pintore, D. J. Walton and T. P. Roberts, A spectral-timing model for ULXs in the supercritical regime, MNRAS 447 (2015) 3243 [1412.4532].

[71] L. Titarchuk and E. Seifina, ESO 243-49 HLX-1: scaling of X-ray spectral properties and black hole mass determination, A\&A 595 (2016) A101 [1609.00780].

[72] S. W. Davis, R. Narayan, Y. Zhu, D. Barret, S. A. Farrell, O. Godet et al., The Cool Accretion Disk in ESO 243-49 HLX-1: Further Evidence of an Intermediate-mass Black Hole, ApJ 734 (2011) 111 [1104.2614].

[73] A. Ptak, E. Colbert, R. P. van der Marel, E. Roye, T. Heckman and B. Towne, Optical Counterparts of Ultraluminous X-Ray Sources Identified from Archival HST WFPC2 Images, ApJS 166 (2006) 154 [astro-ph/0605561].

[74] L. Tao, H. Feng, F. Grisé and P. Kaaret, Compact Optical Counterparts of Ultraluminous X-Ray Sources, ApJ 737 (2011) 81 [1106.0315].

[75] J. C. Gladstone, C. Copperwheat, C. O. Heinke, T. P. Roberts, T. F. Cartwright, A. J. Levan et al., Optical Counterparts of the Nearest Ultraluminous X-Ray Sources, ApJS 206 (2013) 14 [1303.1213].

[76] A. Vinokurov, S. Fabrika and K. Atapin, Optical Counterparts of Ultraluminous X-Ray Sources NGC 4559 X-10 and NGC 4395 ULX-1, ApJ 854 (2018) 176.

[77] A. Vinokurov, S. Fabrika and K. Atapin, Ultra-luminous X-ray sources as supercritical accretion disks: Spectral energy distributions, Astrophysical Bulletin 68 (2013) 139 [1302 . 5630 ].

[78] C. Motch, M. W. Pakull, R. Soria, F. Grisé and G. Pietrzyński, A mass of less than 15 solar masses for the black hole in an ultraluminous X-ray source, Nature 514 (2014) 198 [1410 . 4250].

[79] T. P. Roberts, J. C. Gladstone, A. D. Goulding, A. M. Swinbank, M. J. Ward, M. R. Goad et al., (No) dynamical constraints on the mass of the black hole in two ULXs, Astronomische Nachrichten 332 (2011) 398 [1011.2155].

[80] D. Cseh, F. Grisé, P. Kaaret, S. Corbel, S. Scaringi, P. Groot et al., Towards a dynamical mass of the ultraluminous X-ray source NGC 5408 X-1, MNRAS 435 (2013) 2896 [1308 .1251].

[81] S. Fabrika, Y. Ueda, A. Vinokurov, O. Sholukhova and M. Shidatsu, Supercritical accretion disks in ultraluminous X-ray sources and SS 433, Nature Physics 11 (2015) 551.

[82] P. A. Charles, J. Casares, D. H. P. Jones, T. Broadhurst, P. J. Callanan, D. Carter et al., The bright X ray transient GS2023+338 (=V404 Cyg) in optical outburst and decline, in Two Topics in X-Ray Astronomy, Volume 1: X Ray Binaries. Volume 2: AGN and the X Ray Background (J. Hunt and B. Battrick, eds.), vol. 296 of ESA Special Publication, Nov., 1989. 
[83] R. Soria, D. T. Wickramasinghe, R. W. Hunstead and K. Wu, Measuring the Motion of the Black Hole in GRO J1655-40, ApJL 495 (1998) L95 [astro-ph/ 9801095 ].

[84] G. L. Israel, A. Papitto, P. Esposito, L. Stella, L. Zampieri, A. Belfiore et al., Discovery of a 0.42-s pulsar in the ultraluminous X-ray source NGC 7793 P13, MNRAS 466 (2017) L48 [1609. 06538 ].

[85] M. M. Basko and R. A. Sunyaev, The limiting luminosity of accreting neutron stars with magnetic fields, MNRAS 175 (1976) 395.

[86] A. A. Mushtukov, V. F. Suleimanov, S. S. Tsygankov and J. Poutanen, The critical accretion luminosity for magnetized neutron stars, MNRAS 447 (2015) 1847 [1409.6457].

[87] K. Y. Ekşi, İ. C. Andaç, S. Çıkıntoğlu, A. A. Gençali, C. Güngör and F. Öztekin, The ultraluminous X-ray source NUSTAR J095551+6940.8: a magnetar in a high-mass X-ray binary, MNRAS 448 (2015) L40 [1410 . 5205].

[88] T. Kawashima, S. Mineshige, K. Ohsuga and T. Ogawa, A radiation-hydrodynamics model of accretion columns for ultra-luminous X-ray pulsars, PASJ 68 (2016) 83 [1608 . 04211 ].

[89] S. S. Tsygankov, A. A. Mushtukov, V. F. Suleimanov and J. Poutanen, Propeller effect in action in the ultraluminous accreting magnetar M82 X-2, MNRAS 457 (2016) 1101 [1507. 08288].

[90] F. Pintore, L. Zampieri, L. Stella, A. Wolter, S. Mereghetti and G. L. Israel, Pulsator-like Spectra from Ultraluminous X-Ray Sources and the Search for More Ultraluminous Pulsars, ApJ 836 (2017) 113 [1701.03595].

[91] D. J. Walton, F. Fürst, M. Heida, F. A. Harrison, D. Barret, D. Stern et al., Evidence for Pulsar-like Emission Components in the Broadband ULX Sample, ApJ 856 (2018) 128 [1803. 04424 ].

[92] V. Doroshenko, A. Santangelo and L. Ducci, Searching for coherent pulsations in ultraluminous $X$-ray sources, A\&A 579 (2015) A22 [1410.7264].

[93] K. Belczynski and J. Ziolkowski, On the Apparent Lack of Be X-Ray Binaries with Black Holes, ApJ 707 (2009) 870 [0907.4990]. 